\title{
Dynamics of Foreign Exchange Networks: A Time-Varying Copula Approach
}

\author{
Gang-Jin Wang, ${ }^{1,2}$ Chi Xie, ${ }^{1,2}$ Peng Zhang, ${ }^{1}$ Feng Han, ${ }^{3}$ and Shou Chen ${ }^{1,2}$ \\ ${ }^{1}$ College of Business Administration, Hunan University, Changsha 410082, China \\ ${ }^{2}$ Center of Finance and Investment Management, Hunan University, Changsha 410082, China \\ ${ }^{3}$ China Merchants Bank, Shenzhen 518067, China
}

Correspondence should be addressed to Chi Xie; xiechi@hnu.edu.cn

Received 11 March 2014; Accepted 16 April 2014; Published 6 May 2014

Academic Editor: Fenghua Wen

Copyright (C) 2014 Gang-Jin Wang et al. This is an open access article distributed under the Creative Commons Attribution License, which permits unrestricted use, distribution, and reproduction in any medium, provided the original work is properly cited.

\begin{abstract}
Based on a time-varying copula approach and the minimum spanning tree (MST) method, we propose a time-varying correlation network-based approach to investigate dynamics of foreign exchange (FX) networks. In piratical terms, we choose the daily FX rates of 42 major currencies in the international FX market during the period of 2005-2012 as the empirical data. The empirical results show that (i) the distributions of cross-correlation coefficients (distances) in the international FX market (network) are fat-tailed and negatively skewed; (ii) financial crises during the analyzed period have a great effect on the FX network's topology structure and lead to the US dollar becoming more centered in the MST; (iii) the topological measures of the FX network show a large fluctuation and display long-range correlations; (iv) the FX network has a long-term memory effect and presents a scale-free behavior in the most of time; and (v) a great majority of links between currencies in the international FX market survive from one time to the next, and multistep survive rates of FX networks drop sharply as the time increases.
\end{abstract}

\section{Introduction}

Financial markets are accounted as complex dynamical systems with large quantities of interacting unties $[1,2]$. Financial agents usually interact with each other and their interbehaviors change over time, which means that the interbehaviors are dynamics and widely found in economics and finance $[3,4]$. To capture the interactive behaviors or cross-correlations among heterogeneous entries in financial markets, many scholars generally resort to a powerful analytical tool, namely, correlation network-based methods, which include the minimum spanning tree (MST) approach proposed by Mantegna [5], the correlation threshold methods developed by Boginski et al. [6] and Onnela et al. [7], and the approach of planar maximally filtered graph (PMFG) designed by Tumminello et al. [8]. The network analysis idea has been widely applied in financial markets, such as stock markets [9-14] and foreign exchange (FX) market [1520]. Among correlation network-based approaches, the MST method is often preferred because of its robustness and simplicity [10]. However, the MST method and its improvements ignore the volatiles and nonlinearities of financial time series. That is to say, they cannot really detect the dynamic interbehaviors among different financial agents in financial markets. Therefore, the purpose of this paper is to propose a dynamic correlation network-based approach by combining a time-varying copula method and the MST approach for studying the dynamic topology and market natures of financial networks. In practical terms, we focus our study on networks' dynamics of the international FX market because it is the biggest and most liquid financial market where foreign currencies are traded [21].

The motivations that led us to combine the two aforementioned methods to investigate dynamics of FX networks can be summed up as follows. On the one hand, the MST and its improvements are usually used to identify the clustering behavior and dominant currencies in the FX network. To examine the dynamic behavior of the network, pervious works often employ a rolling window analysis, such as $[10$, 22]. However, some drawbacks are found in the dynamic 
MSTs using a rolling window analysis as follows. (i) The choice of parameters of the rolling window is dependent on the scholars' preference; that is, the window width and step length are selected arbitrarily [23]. For example, Onnela et al. [10] set the size of window as four calendar years (approximately 1000 trading days), while Song et al. [22] fix the length of window as 1250 trading days. (ii) In the MST method, the cross-correlation coefficient between two financial units is usually measured by the Pearson's correlation coefficient (PCC) that is a linear correlation method. Nevertheless, the PCC cannot quantify the nonlinear relationship between two heterogeneous entries [21] and ignores financial variables' volatile and nonnormality features [24]. It should be noted that some works attempt to apply other dynamic approaches in the MST. For example, Lyócsa et al. [23] employ the dynamic conditional correlations (DCC) multivariate generalized autoregressive conditional heteroscedasticity (MVGARCH) approach to construct the MST for the US stock market. Trancoso [25] uses the Baba-Engle-Kraft-Kroner (BEKK) model to develop the conditional correlation matrix and apply it in the dynamic network analysis. However, Lyócsa et al. [23] and Trancoso [25] assume that the financial variables obey the normal distribution and neglect their nonnormality characteristics.

On the other hand, copula methods proposed by Sklar $[26,27]$ are a widely useful tool for deriving joint distributions given the marginal distributions, especially when the variables follow nonnormal distributions [28]. Besides, copulas can be employed to investigate the dependence beyond the linear cross-correlation by the PCC and allow for a timevarying nonlinear analysis. Moreover, copulas can be supplied as a powerful analytical instrument to measure dynamic dependence structures between financial variables. In a word, copulas have better properties and more advantages (see $[29,30])$ than the traditional linear correlation methods and attract many scholars to use them in various finance applications [31-37]. For instance, Patton [35] constructs time-varying copula models to examine the dependence between two FX rates, that is, Deutsche Mark/US Dollar (DM/USD) and Japanese Yen/US Dollar (JPY/USD). He also makes a comparison between copulas and GARCH models and finds that the former can better describe the dependence or cross-correlation of FX rates than the latter. Diks et al. [36] use several copula models to analyze the dependence among five currencies (i.e., Canadian Dollar (CAD), Swiss Franc (CHF), European Euro (EUR), British Pound (GBP), and JPY) against USD and show that the Student's $t$-copula model is evidently superior to the Gaussian, Gumbel, and Clayton copula models. Dias and Embrechts [37] investigate the dependence between EUR/USD and JPY/USD from October 1, 2000, to October 1, 2008, by employing the time-varying copula-GARCH models. Their empirical results suggest that the dependence between the two currencies is dynamic and a time-varying copula approach with given correlation specifications has better outcomes than some conventional dynamic methods (e.g., BEKK).

In consideration of the above-mentioned motives, based on a time-varying copula approach and the MST method, we aim to construct time-varying FX networks and analyze their topological dynamics and market properties. We choose 42 major currencies' daily FX rate series in the international FX market during the years 2005-2012 as the empirical data. In empirical process, we first use a time-varying copula to calculate the dynamic cross-correlation coefficients $\rho_{t}$ among different currencies. More specifically, we adopt an $\operatorname{AR}(p)$ GARCH(1,1)- $t$ model to characterize the marginal distribution of returns for FX rates and then estimate the dependence parameters of the time-varying Student's $t$-copula model and obtain time-varying cross-correlation coefficients. Next, on the basis of time-varying cross-correlation coefficients, we construct time-varying cross-correlation matrices (CMs) $\mathrm{C}_{t}$ for 42 major currencies in the international FX market. Then, we transform the time-varying CMs into time-varying FX networks by the MST approach. Finally, we examine topological dynamics and statistic features for time-varying FX networks.

The remainder of the paper is organized as follows. Section 2 represents the empirical data. A time-varying correlation network-based approach by combining a timevarying copula model with the MST method is described in Section 3. In Section 4, the time-varying FX networks are constructed and main empirical results are showed. Some conclusions are drawn in Section 5.

\section{Data Set}

As for the empirical data set, we choose the daily FX rates of 42 major currencies in the international FX market from January 4, 2005, to December 31, 2012. Following Jang et al. [18] and Wang et al. [21], we select the special drawing right (SDR) as the numeraire. The 42 currencies are from 7 different continents or regions. Their detailed information is showed as follows: (1) Africa: Egyptian Pound (EGP) and South African Rand (ZAR); (2) Asia: Chinese Renminbi (CNY), Indian Rupee (INR), Indonesian Rupiah (IDR), Japanese Yen (JPY), Malaysian Ringgit (MYR), Pakistani Rupee (PKR), Philippines Peso (PHP), Singapore Dollar (SGD), South Korean Won (KRW), Taiwanese Dollar (TWD), Thai Baht (THB), and Vietnamese Dong (VND); (3) Europe: British Pound (GBP), Czech Koruna (CZK), European Euro (EUR), Hungarian Forint (HUF), Icelandic Krona (ISK), Norwegian Krone (NOK), Polish Zloty (PLN), Romanian New Leo (RON), Russian Rubles (RUB), Swedish Krona (SEK), Swiss Franc (CHF), and Turkish New Lira (TRY); (4) Latin America: Argentine Peso (ARS), Brazilian Real (BRL), Chilean Peso (CLP), Colombian Peso (COP), Peruvian New Sole (PEN), and Mexican Peso (MXN); (5) Middle East: Bahrain Dinar (BHD), Israeli New Shekel (ILS), Jordanian Dinar (JOD), Kuwaiti Dinar (KWD), Saudi Arabian Riyal (SAR), and United Arab Emirates Dirham (AED); (6) North America: Canadian Dollar (CAD) and US Dollar (USD); (7) Pacific Ocean: Australian Dollar (AUD) and New Zealand Dollar (NZD). All the FX rates are obtained from the website of the Pacific Exchange Rate Service (http://fx.sauder.ubc.ca/data.html). We define the return of currency $i$ on day $t$ as $r_{i, t}=100\left(\ln P_{i, t}-\ln P_{i, t-1}\right)$, where $P_{i, t}$ is the daily FX rate of currency $i$ on day $t$. During the analyzed period, each currency's returns have 2003 observations. 


\section{Methodology}

In this section, we first introduce the time-varying copula model including the model for marginal distributions, the dynamic Student's $t$-copula model, and the estimation of parameters. After that, we propose the time-varying correlation network-based approach by the MST method.

3.1. Model for Marginal Distributions. Following Patton [35] and Dias and Embrechts [37], we use an $\operatorname{AR}(p)-\mathrm{GARCH}(1,1)-$ $t$ model, which considers the influences of asymmetric information, to characterize the returns' marginal distributions of currency $i$. The proposed model is defined as follows:

$$
\begin{gathered}
r_{i, t}=\mu+\sum_{j=1}^{p} \phi_{j} r_{i, t-j}+\varepsilon_{i, t}, \\
\varepsilon_{i, t}=\sigma_{i, t} z_{i, t}, \quad z_{i, t} \sim t(\nu), \\
\sigma_{i, t}^{2}=\omega_{i}+\alpha_{i} \varepsilon_{i, t-1}^{2}+\beta_{i} \sigma_{i, t-1}^{2},
\end{gathered}
$$

where $\phi_{j}$ are autoregressive (AR) coefficients, $z_{i, t}$ obeys a Student's $t$-distribution, $v$ is the degree of freedom, and $\sigma_{i, t}^{2}$ is the conditional variance of $\varepsilon_{i, t}$ with the following parameter restrictions: $\omega_{i}>0, \alpha_{i}>0, \beta_{i}>0$, and $\alpha_{i}+\beta_{i}<1$. To be simple and effective enough, we use an $\operatorname{AR}(1)$ process.

3.2. The Dynamic t-Copula Model. According to Diks et al. [36] and Dias and Embrechts [37], the dynamic $t$-copula model has a better capability for quantifying dynamic correlations in the FX rates data compared with Gaussian, Gumbel, and Clayton copula models. Therefore, in this paper, we employ the dynamic Student's $t$-copula model to capture time-varying cross-correlations in the international FX market. Let us briefly show the dynamic Student's $t$-copula model as follows.

For all $u_{t}, v_{t} \in[0,1]$, the density of dynamic Student's $t$ copula is defined by

$$
\begin{aligned}
c_{t}\left(u_{t}, v_{t} \mid \theta_{t}, n\right) & \\
= & \frac{1}{\sqrt{1-\theta_{t}^{2}}} \frac{\Gamma((n+2) / 2) \Gamma(n / 2)}{[\Gamma((n+1) / 2)]^{2}} \\
& \times\left[1+\left(T_{n}^{-1}\left(u_{t}\right)^{2}+T_{n}^{-1}\left(v_{t}\right)^{2}-2 \theta_{t} T_{n}^{-1}\left(u_{t}\right) T_{n}^{-1}\left(v_{t}\right)\right)\right. \\
& \left.\times\left(n\left(1-\theta_{t}^{2}\right)\right)^{-1}\right]^{-(n+2) / 2} \\
& \times\left[\left(1+\frac{T_{n}^{-1}\left(u_{t}\right)^{2}}{n}\right)\left(1+\frac{T_{n}^{-1}\left(v_{t}\right)^{2}}{n}\right)\right]^{(n+1) / 2},
\end{aligned}
$$

where $T_{n}^{-1}(\cdot)$ represents the inverse of the cumulative distribution function (CDF) of the Student's $t$-distribution with $n$ degrees of freedom [39], $\theta_{t} \in(0,1)$ denotes the linear correlation coefficient, and $\Gamma(\cdot)$ is the Gamma function.
As proposed in [31], the time-varying dependence coefficients of the Student's $t$-copula is defined as

$$
\rho_{t}=\widetilde{\Lambda}\left(\gamma_{0}+\gamma_{1} \rho_{t-1}+\gamma_{2} \frac{1}{10} \sum_{j=1}^{10} T_{n}^{-1}\left(u_{t-j}\right) T_{n}^{-1}\left(v_{t-j}\right)\right) \text {, }
$$

where $\widetilde{\Lambda}(x)=\left(1-e^{-x}\right) /\left(1+e^{x}\right)$ is the modified logistic function, which can guarantee that cross-correlation coefficients retain in the interval $(-1,1)$ at all times; $\gamma_{k}(k=0,1,2)$ are unknown parameters.

3.3. Estimation of Copula Parameters. Following Wang et al. [34] and Lai et al. [40], we adopt the inference-functionfor-margins (IFM) method rather than the exact maximum likelihood method to estimate the copula parameters, because the former needs less computation than the latter. The IFM approach proposed by Joe and $\mathrm{Xu}$ [41] is a two-step estimation, which can be used to estimate the parameters of marginal distributions and the copula functions separately. For more detailed advantages, see [34, 41]. The procedure of IFM is showed as follows.

Step 1. The marginal parameters are estimated by the maximum likelihood (ML) as

$$
\widehat{\xi}_{i}=\arg \max \sum_{t=1}^{T} \ln f_{i, t}\left(z_{i, t} \mid \Omega_{t-1} ; \xi_{i}\right),
$$

where $f_{i, t}(\cdot \mid \cdot)$ denotes the conditional marginal density of currency $i$ at time $t, \xi_{i}$ is the marginal parameter of returns of currency $i$, and $\Omega_{t-1}$ is the past information set.

Step 2. Given $\xi_{i}$, suppose we have $\xi_{u}$ and $\xi_{v}$; the copula parameters can be estimated as

$$
\begin{array}{r}
\widehat{\xi}_{c}=\arg \max \sum_{t=1}^{T} \ln c_{t}\left(F_{u, t}\left(z_{u, t} \mid \Omega_{t-1} ; \widehat{\xi}_{u}\right),\right. \\
\left.F_{v, t}\left(z_{v, t} \mid \Omega_{t-1} ; \widehat{\xi}_{v}\right) ; \xi_{c}\right) .
\end{array}
$$

3.4. The Time-Varying Correlation Network-Based Approach. After obtaining the time-varying cross-correlation coefficients between any two currencies by a time-varying copula approach, we can build $N \times N$ time-varying cross-correlation matrices $(\mathrm{CMs}) \mathbf{C}_{t}$ with elements $\rho_{t}(i, j)$ for currencies $i$ and $j$, where $1 \leq i$ and $j \leq N$ (in our case, $N=42$ ). According to the idea of MST proposed by Mantegna [5], we transform time-varying CMs into the corresponding distance matrices $\mathbf{D}_{t}$ by a distance measure $d_{t}(i, j)=\sqrt{2\left(1-\rho_{t}(i, j)\right)}$ that falls in the interval $[0,2]$ and meets the three axioms of the Euclidean distance. On the basis of time-varying distance matrices $\mathbf{D}_{t}$, we can obtain time-varying networks for studying the international FX market by using the Kruskal's algorithm [42], that is, time-varying MSTs link $N$ currencies with $N-1$ edges. At each time $t$, the MST network extracts the most important information (e.g., the strongest cross-correlations among currencies) in the international FX market. The proposed time-varying correlation network-based approach 
can be used to examine dynamics of the international FX market over time.

To investigate dynamics of FX networks, we introduce some topological measures as follows. We use a quantity of average path length (APL) to quantify the MST network's density [43], which is defined by

$$
\mathrm{APL}_{t}=\frac{2}{N(N-1)} \sum_{i=1, j>i}^{N} l_{i j}^{t}
$$

where $l_{i j}^{t}$ is the length of the shortest path between two vertexes (currencies) $i$ and $j$ at time $t$ [21].

The measure of mean occupation layer (MOL) proposed by Onnela et al. $[9,10]$, which can be employed to analyze the spread of nodes on the MST and characterize the density changes of the network, is defined as

$$
\operatorname{MOL}_{t}\left(v_{c}\right)=\frac{1}{N} \sum_{i=1}^{N} \operatorname{lev}\left(v_{i}^{t}\right),
$$

where $v_{c}$ is the central vertex of the MST at time $t$ and $\operatorname{lev}\left(v_{i}^{t}\right)$ defines the level of vertex $v_{i}$ with reference to $v_{c}$, whose level is set as zero.

We introduce a concept of maximum degree $k_{\max }$, which is defined as the number of linkages of the central vertex in the MST $[21,43]$.

The scale-free behavior is widely found in different networks $[10,15,21,44,45]$. The scale-free network is such that the degree distribution of the network has a power-law tail; that is,

$$
P(k) \sim k^{-\alpha},
$$

where $P(k)$ is the distribution function of vertex degrees $k$, and $\alpha$ is the exponent. We adopt a powerful tool developed by Clauset et al. [38] to estimate the power-law exponent and the corresponding $P$ value. This tool combines ML fitting methods with goodness-of-fit tests using the KolmogorovSmirnov statistic and likelihood ratios.

\section{Empirical Results}

4.1. Statistics of Cross-Correlation Coefficients and Distances of MST. Before studying dynamics of FX networks, we first analyze statistical properties of cross-correlation coefficients and distances of MST for 42 currencies in the international FX market. The cross-correlation coefficient series contains $N(N-1) / 2$ observations, at each time, while the distance set of MST only contains the $N-1$ most important links. In Figures 1 and 2, we present the time evolution graphs for four descriptive statistics (mean, standard deviation, skewness, and kurtosis) of cross-correlation coefficients and distances of MST, respectively. From each figure, it can be found that the four descriptive statistics vary over time and have a high volatile during the US subprime crisis and the 2008 world financial crisis. Especially in the period of June 2007 to July 2009, the international FX market (network) has stronger cross-correlations or smaller distances among
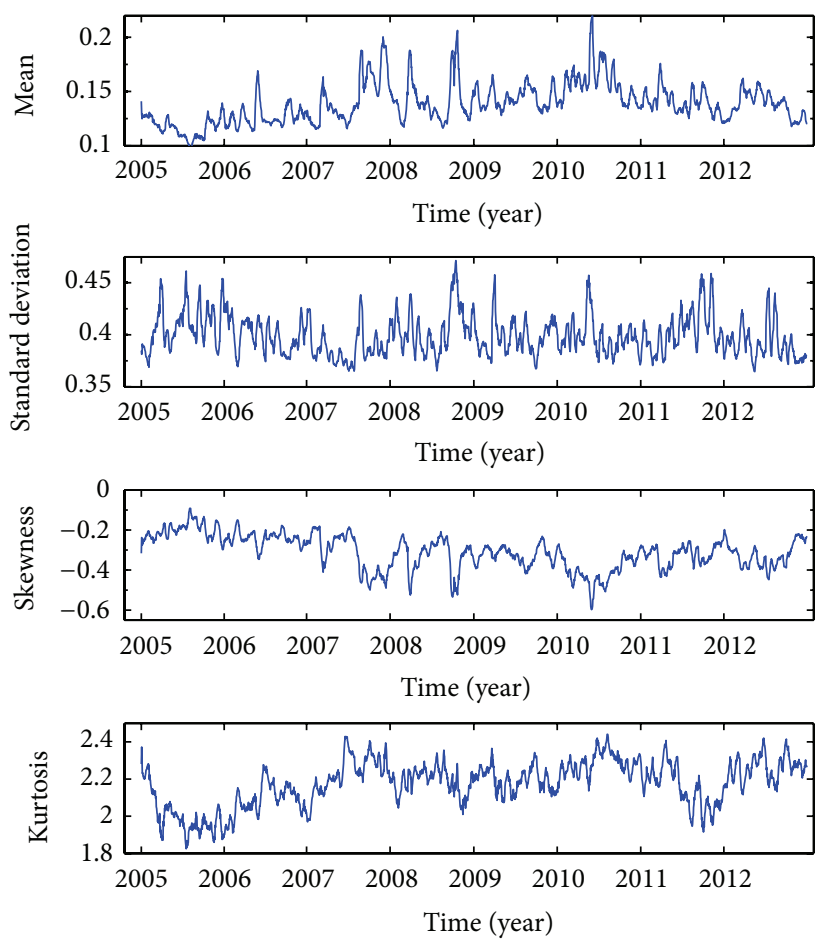

FIGURE 1: The mean, standard deviation, skewness, and kurtosis of cross-correlation coefficients of 42 currencies in the international FX market as functions of time.
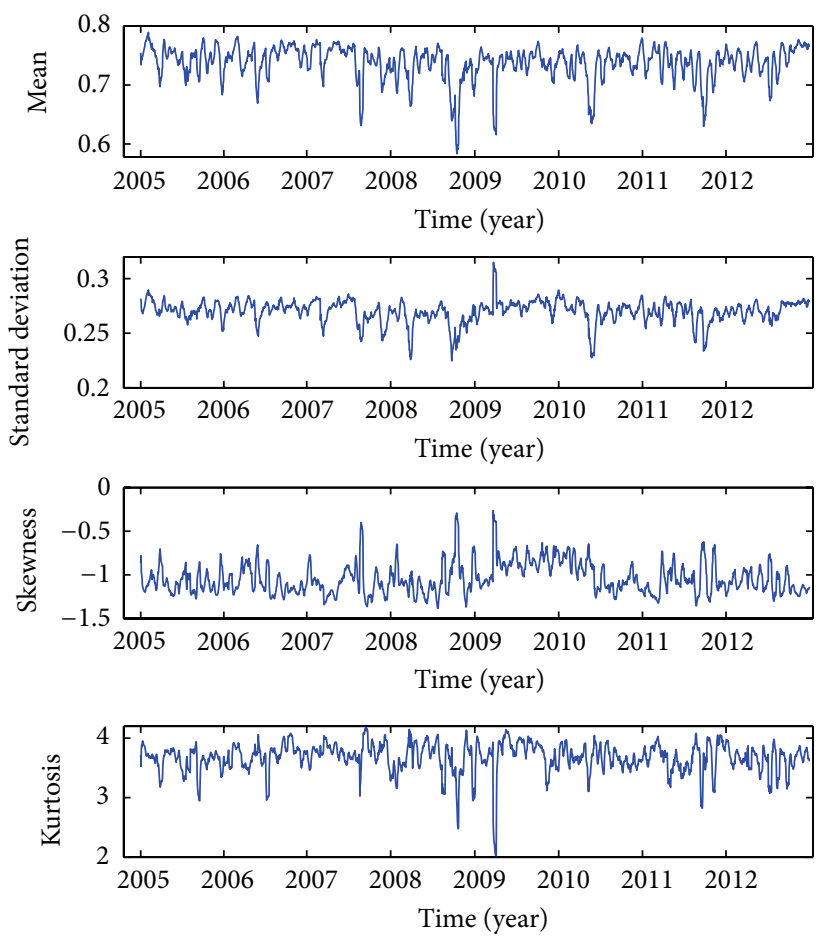

FIGURE 2: The mean, standard deviation, skewness, and kurtosis of distances of MST of 42 currencies in the international FX market as functions of time. 


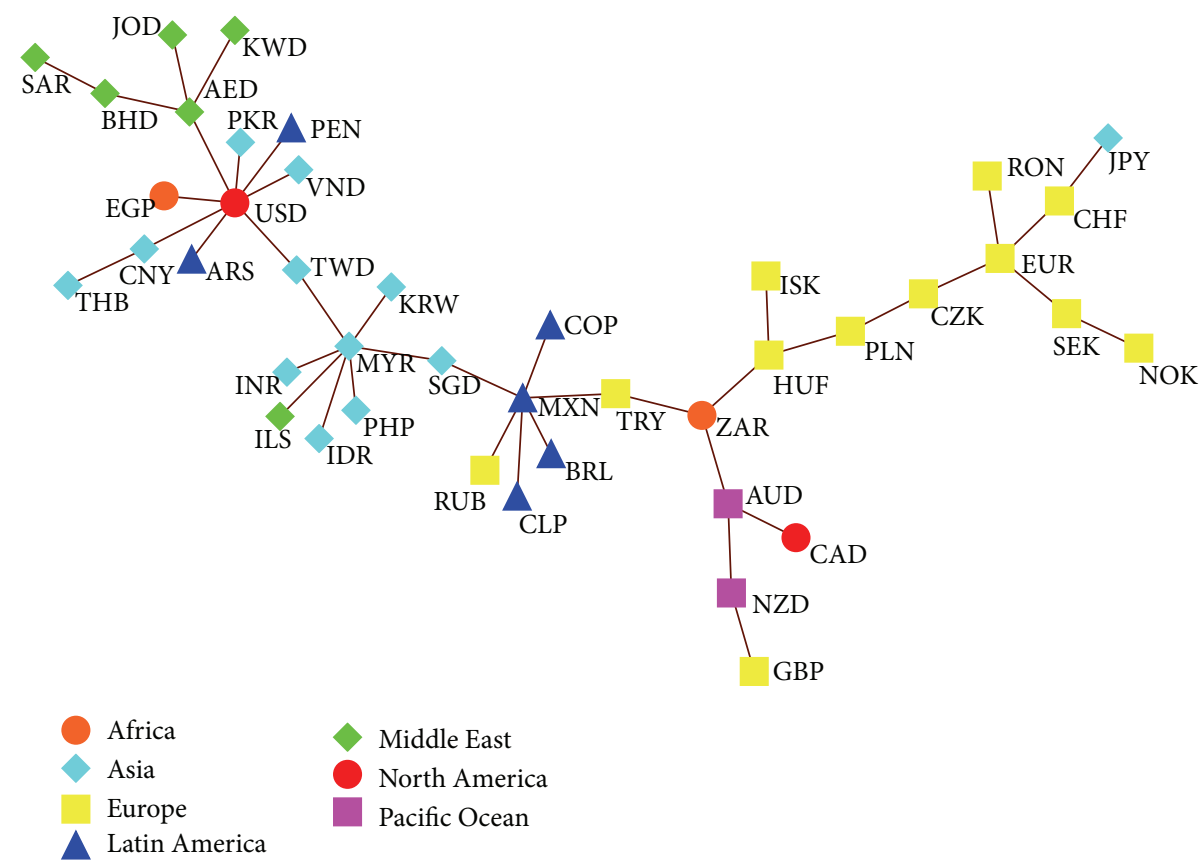

FIGURE 3: MST of 42 currencies in the international FX market on January 5, 2005, as a representative of the period of before financial crises.

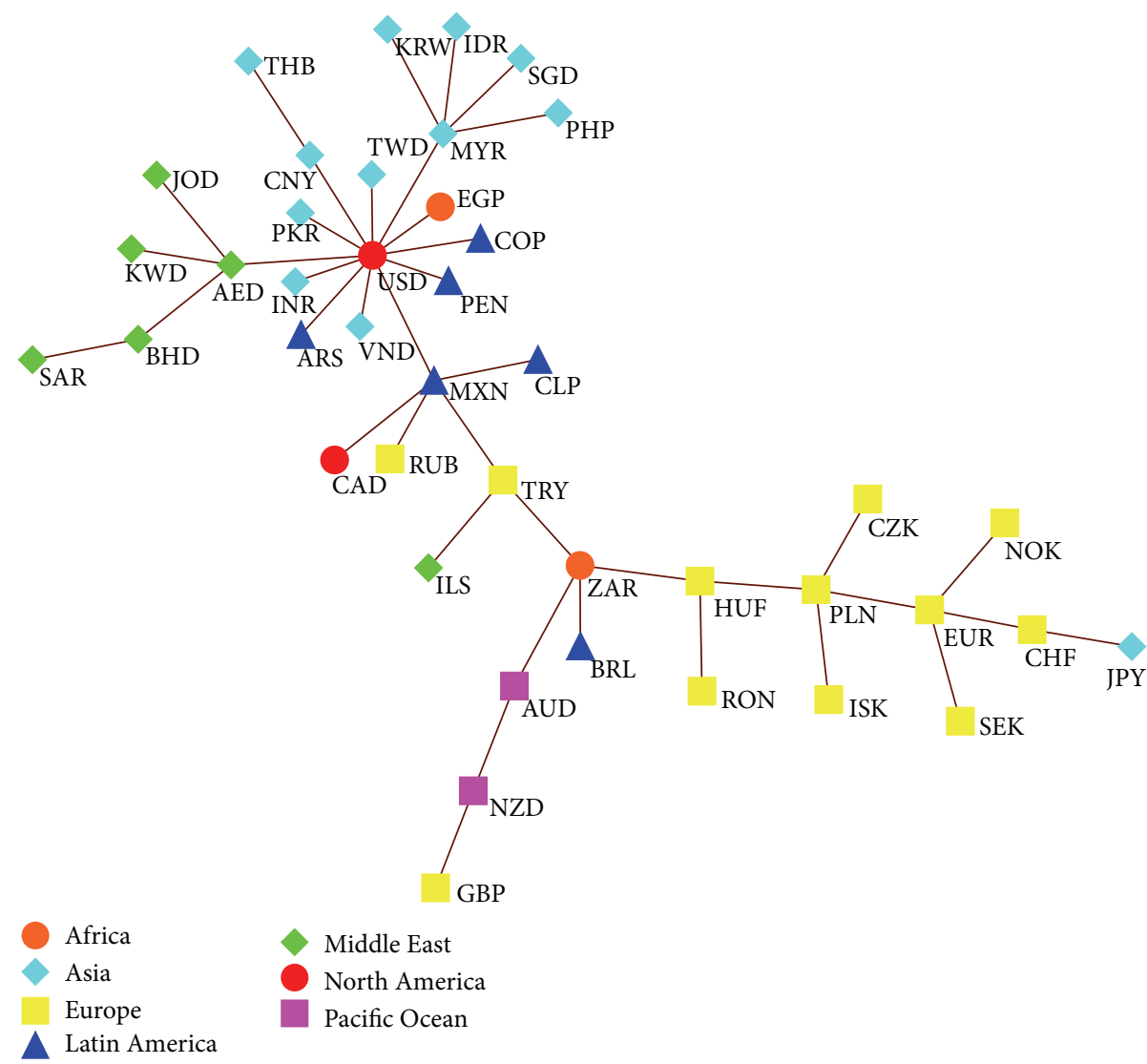

FIGURE 4: MST of 42 currencies in the international FX market on January 2, 2008, as a representative of the period of during financial crises. 


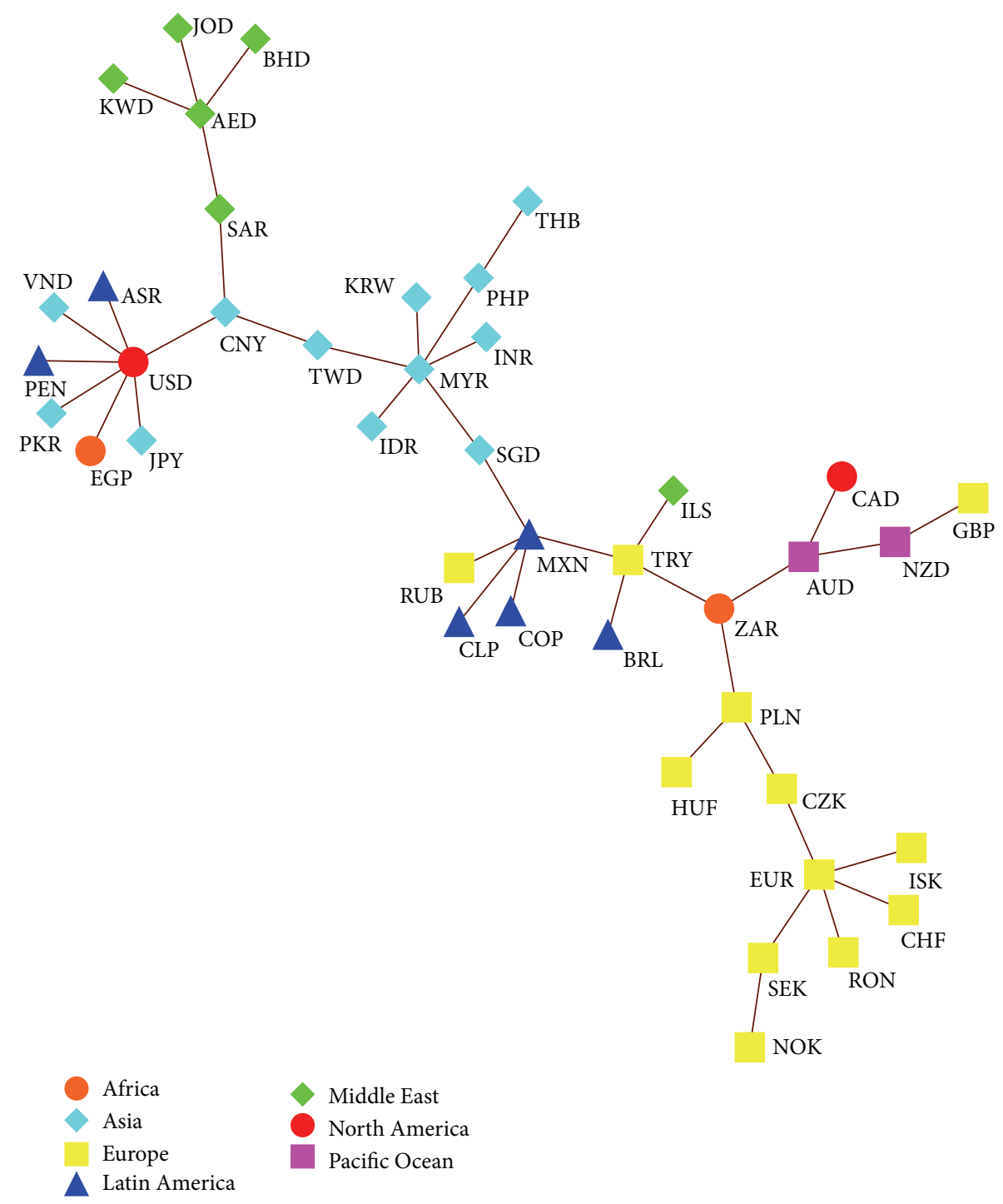

FIGURE 5: MST of 42 currencies in the international FX market on January 3, 2012, as a representative of the period of after financial crises.

currencies than other periods. This phenomenon confirms the proposal in [46] that the financial crisis often causes an increase of the market's cross-correlations. As shown in Figures 1 and 2, it can be found that the values of skewness of cross-correlation coefficients and distances at any time are less than 0 , while values of kurtosis for most of time are not equal to 3 . This finding implies that the distributions of crosscorrelation coefficients (distances) in the international FX market (network) are fat-tailed and negatively skewed.

4.2. MST Results. Considering that financial crises have a strong influence on the international FX market, we choose three days (i.e., January 5, 2005; January 2, 2008; and January 3,2012 ) as representatives of three periods of before, during, and after financial crises. We present the three MSTs of 42 currencies in the international FX market in Figures 3, 4, and 5 , respectively. In each MST figure, currencies from the same continent (region) are marked with the same color and shape.
From Figure 3, which demonstrates the situation before financial crises, one can find that most of currencies are gathered together according to geographical distributions, such as the European cluster, Asian cluster, Middle Eastern cluster, and Latin American cluster with EUR, MYR, AED, and MXN at their centers, respectively. In the FX network, the most important cluster is the international cluster with USD at its hub, which is directly or indirectly connected with currencies from Asia, Middle East, Latin America, and Africa. This outcome shows that USD is the predominant world currency. An interesting cluster is composed of GBP from Europe, NZD and AUD from Pacific Ocean, CAD from North America, and ZAR from Africa. We denote this cluster as the Commonwealth cluster because countries of the five currencies are members of the Commonwealth of Nations. In the MST network, we find that three major currencies in the international FX market, namely, EUR, CHF, and JPY are linked together. 
As illustrated in Figure 4, during the global financial crisis, it can be observed that a lot has changed in the FX network. Notable changes are that USD becomes more centered in the MST, and the Latin American cluster and Asian cluster almost broke and their currencies directly or indirectly shift to USD. That is to say, during the financial crisis, most currencies from Asia, Middle East, and Latin America are tightly linked to USD, which indicates that the financial crisis can lead to a huge comovement effect among currencies in the international FX market. Although the European cluster and the Commonwealth cluster still remain in the network, their structure and currencies' position changed as a result of the influence by the financial crisis.

Compared with the MSTs in Figures 3 and 4, as drawn in Figure 5, the FX network recovered to the precrisis state but its structure and currencies' position have a lot of changes. For instance, the Asian cluster and Latin American cluster are formed again. At this point, the Commonwealth cluster has reappeared in the network with the same structure and position of their currencies as they appeared in Figure 3. One can see a remarkable change that JPY deviates from the European cluster and connects to the international cluster with USD at its centre. It is interesting to note that CNY links with USD, TWD, and SAR. One possible interpretation of the linkages is that US, Taiwan, and Saudi Arabia are China's important and top trading partners.

From Figures 3, 4, and 5, we can obtain some conclusions as follows: (i) USD is the predominant world currency and has a powerful influence in the monetary system. (ii) The European cluster has a relatively stable structure and this may be ascribed to the influence of EUR. (iii) Currencies from the Middle East except for ILS always form a cluster and link to USD. Possible explanations are that Saudi Arabia, the United Arab Emirates, Kuwait, Jordanian, and Bahrain are oil-producing countries (the former three countries are members of the Organization of the Petroleum Exporting Countries) and have a mass of USD holdings, and most of their currencies peg to USD. (iv) The Commonwealth cluster is formed in the FX network, suggesting that the Commonwealth nations maybe have the same currency mechanism.

4.3. Dynamics of Topological Features. In this subsection, we aim to investigate the dynamical evolution of time-varying FX networks' topological features. To begin with it, we show the calculation results of the average path length (APL), mean occupation layer (MOL), and maximum degree $k_{\max }$ in Figure 6. As for the density measures of APL and MOL, both of their patterns do not show any tendency but with a fluctuation above and below. The values of maximum degree $k_{\max }$ also have a large volatility, especially during the period of financial crises. Then, we estimate the power-law exponent and the corresponding $P$ value for each MST and present their outcomes in Figure 7. The estimated power-law exponent also changes over time and varies from 2.09 to 3.5 . Although a handful of (about 309) $P$ values are less than 0.1, the power-law hypothesis can be accepted for most MSTs. This finding suggests the FX network is a scale-free network
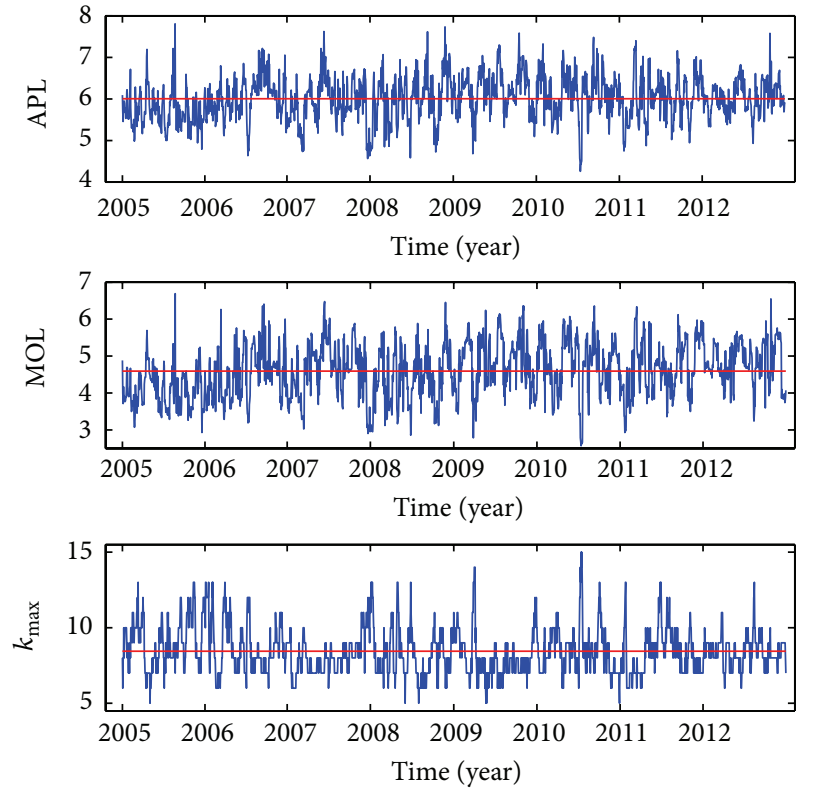

FIGURE 6: The average path length (APL), mean occupation layer (MOL), and maximum degree kmax of MST of 42 currencies in the international FX market as functions of time. In each panel, the red solid line stands for the corresponding statistical average value over the time investigated.
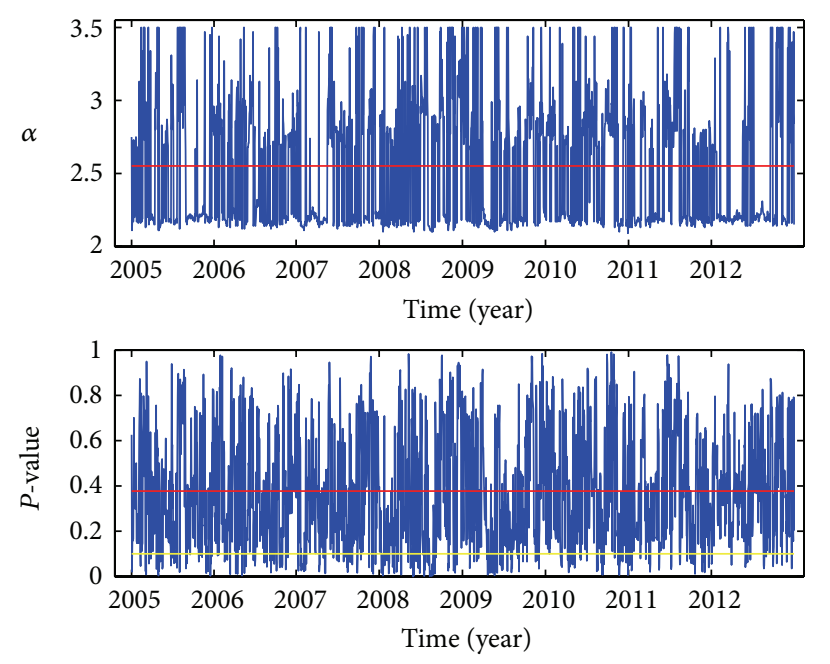

FIGURE 7: The estimated power-law exponent $\alpha$ and the corresponding $P$ value of degree distribution of MST of 42 currencies in the international FX market as functions of time. In each panel, the red solid line stands for the corresponding statistical average value over the time investigated. In the bottom panel, the yellow solid line represents the value of 0.1 . As proposed in [38], if the $P$ value is greater than 0.1 , the power-law hypothesis is accepted for the investigated data; otherwise it is rejected.

in most of the time. That is, a small number of vertexes (currencies, such as USD) always have the vast majority of connections, while most of vertexes have a very few links.

Similar to Qiu et al. [47], we further examine dynamics of topological features of FX networks by analyzing the time correlations. In practical terms, we employ the detrended 


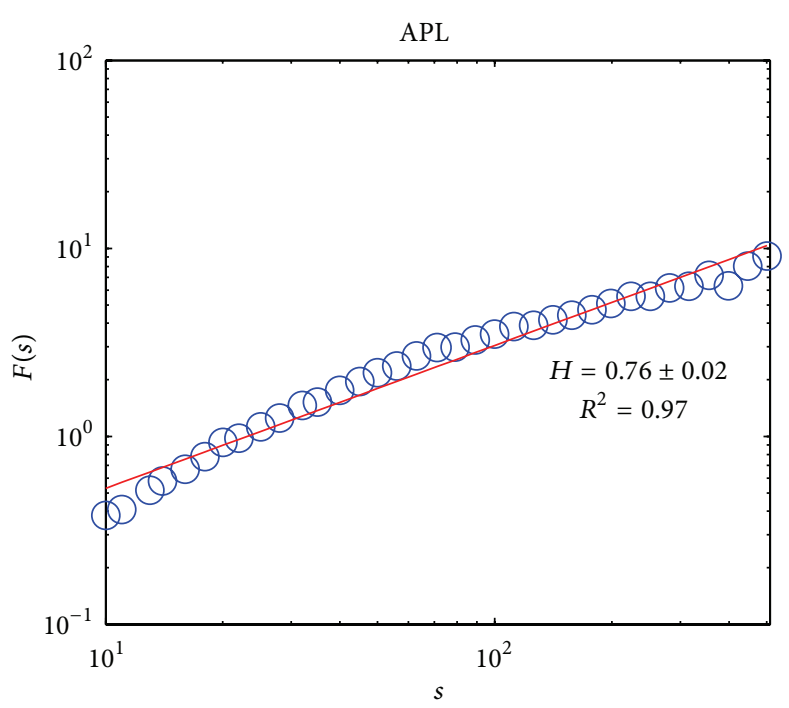

(a)

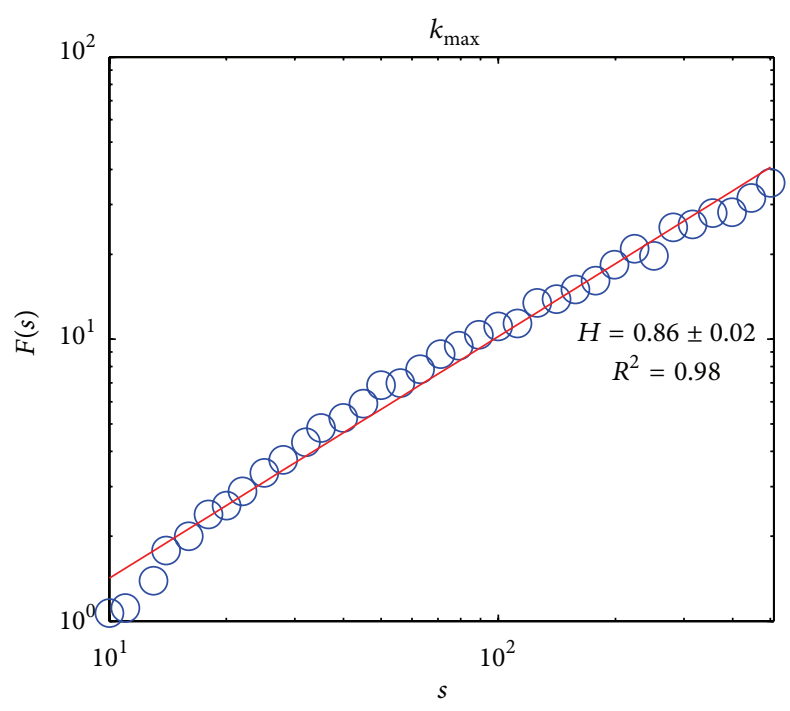

(c)

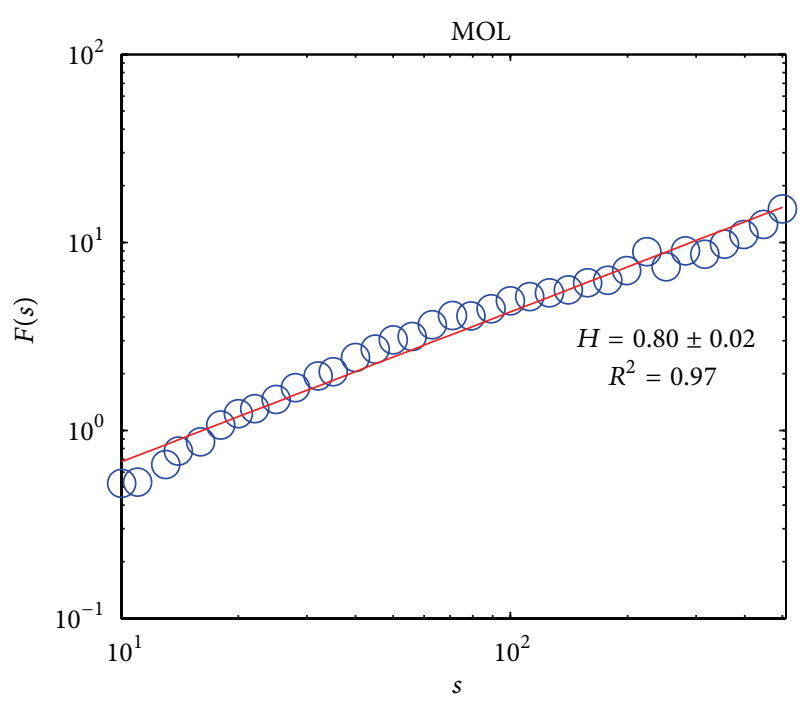

(b)

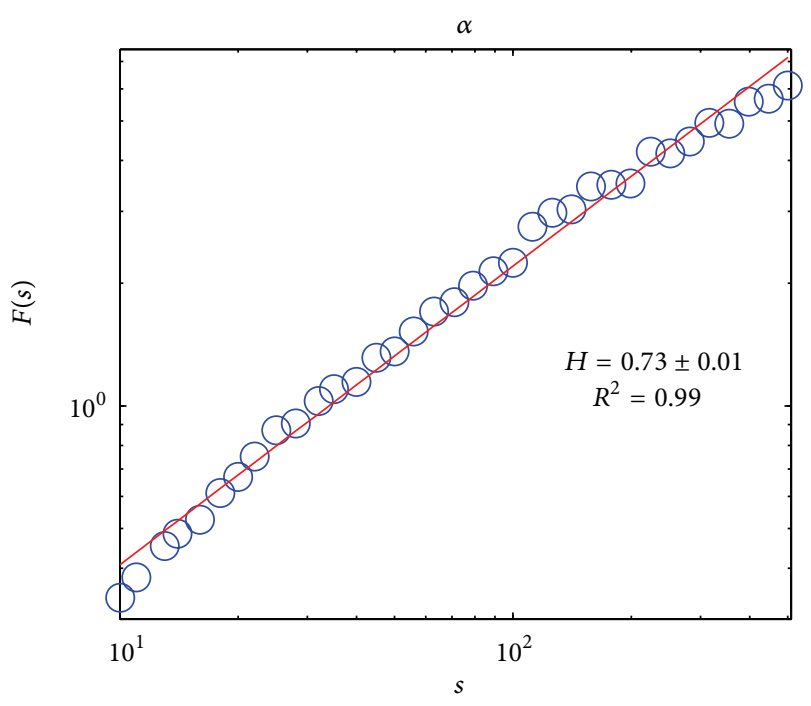

(d)

FIGURE 8: The DFA functions of the average path length (APL), mean occupation layer (MOL), and maximum degree $k_{\max }$ and the estimated power-law exponent $\alpha$ on log-log plots. In each panel, the red solid line stands for the corresponding linear fitting curve, and the estimated Hurst exponent $H$ and its corresponding coefficient of determination $R^{2}$ are presented. The Hurst exponent $0.5<H<1.0$ implies that the time series is long-range correlated or has a long-term memory.

fluctuation analysis (DFA) method proposed by Peng et al. [48], which can be used to quantify long-range correlations of a nonstationary time series. The DFA approach provides a relationship between the DFA function $F(s)$ and the time scale $s$, characterized by a power-law $F(s) \sim s^{-H}$, where $H$ is the well-known Hurst exponent. The Hurst exponent $H=0.5,0<H<0.5$, and $0.5<H<1.0$ means uncorrelated, long-term correlated, and anticorrelated time series, respectively. The DFA functions of the APL, MOL, maximum degree $k_{\max }$, and the estimated power-law exponent are drawn in Figure 8. We calculate the Hurst exponents for APL, MOL, $k_{\max }$, and the power-law exponent as $0.76 \pm 0.02,0.80 \pm$ $0.02,0.86 \pm 0.02$, and $0.73 \pm 0.01$, respectively, which are all larger than 0.5 . These results mean that the four topological measures are long-range correlated and thus suggest that the FX network has a long-term memory effect.

4.4. Single- and Multistep Survival Rates. In order to study the robustness of links over time and the long-term evolution of FX networks, respectively, we use two measures, that is, the single-step survival rate (SSR) and the multistep survival ratio (MSR) proposed by Onnela et al. $[9,10]$. The measure of SSR is defined as the fraction of links found in two consecutive MST at times $t$ and $t+1$; that is,

$$
\operatorname{SSR}(t)=\frac{1}{N-1}|E(t) \cap E(t+1)|,
$$




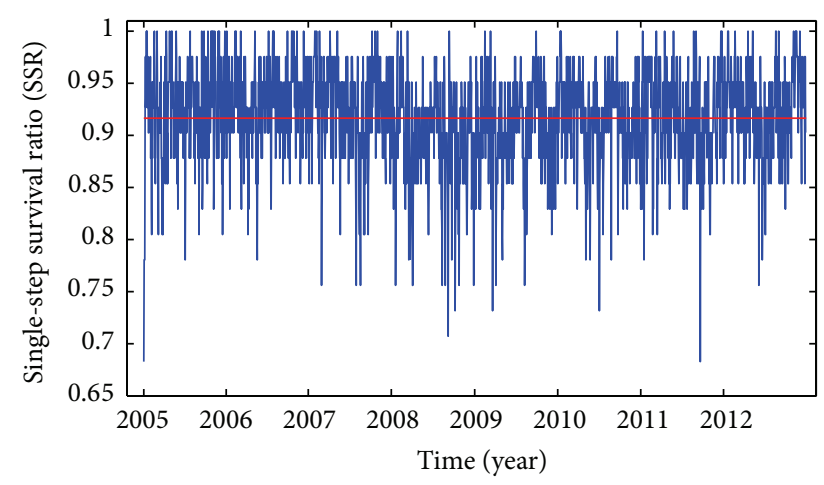

FIgURE 9: The single-step survival ratio (SSR) of MST of 42 currencies in the international FX market as a function of time. The red solid line stands for the corresponding statistical average value over the time investigated.

where $E(t)$ represents the set of edges of the MST at time $t$, $\cap$ is the intersection operator, and $|\cdots|$ gives the number of elements in the set [10]. The MSR measure is defined by

$$
\begin{gathered}
\operatorname{MSR}\left(t_{0}, \delta\right)=\frac{1}{N-1} \mid E\left(t_{0}\right) \cap E\left(t_{0}+1\right) \cdots E\left(t_{0}+\delta-1\right) \\
\cap E\left(t_{0}+\delta\right) \mid,
\end{gathered}
$$

where $t_{0}$ stands for the initial time and $\delta$ is the step length.

In Figure 9, we plot the time-varying SSRs for the MST. The mean value of SSR is close to 0.92 , which shows that a great majority of links between currencies in the international FX market survive from one time to the next. Moreover, we find that about 80 SSRs are equal to 1, indicating that the two consecutive networks at times $t$ and $t+1$ are identical. We also investigate the time correlations of the SSR series by the DFA method and present the results in Figure 10. One can find that the Hurst exponent for the SSR series is $0.68 \pm 0.01$, once again suggesting that the long-range memory effect exists in the FX network. In Figure 11, we show the MSR of MST of 42 currencies in the international FX market as a function of time for different initial time $t_{0}$. In Figure 11, the initial time $t_{0}$ is the first trading date of the year, and 8 curves of MSR are presented. For each curve of MSR, it drops rapidly as the time increases, which implies that the long-term stability of the FX network is falling as the time is increasing. However, we also find that each MSR is usually unchanged and moves toward a constant in the last or middle period of time, meaning that some structures or clusters (e.g., the Middle Eastern cluster) of the FX network are always preserved and stabilized.

\section{Conclusions}

In this paper, we investigate the daily FX rates of 42 major currencies in the international FX market during the period of 2005-2012 and construct time-varying FX networks by a time-varying copula approach and the MST method. In detail, we first use the $\operatorname{AR}(p)-\mathrm{GARCH}(1,1)-t$ model to characterize the returns' marginal distributions of FX rates. Then

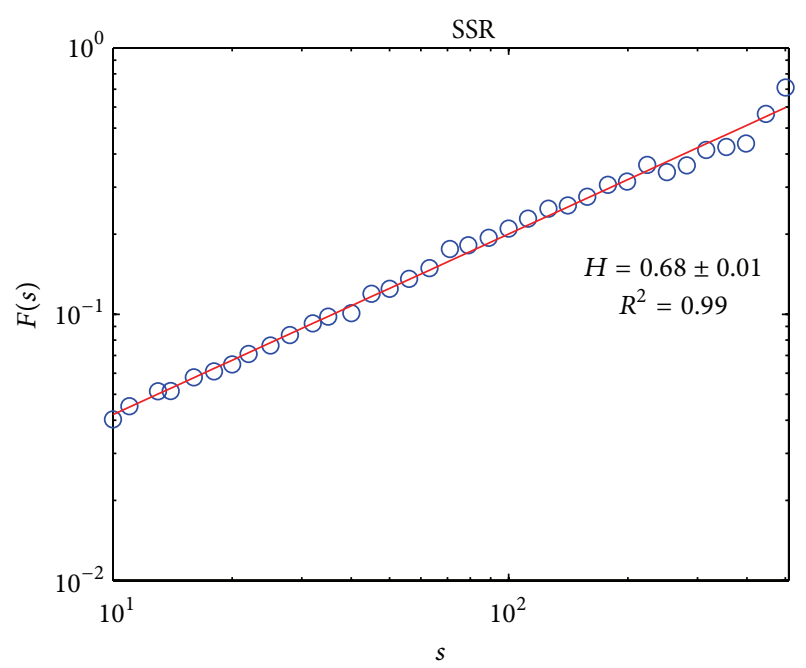

FIGURE 10: The DFA function of the single-step survival ratio (SSR) on a log-log plot. The red solid line stands for the associated linear fitting curve, and the estimated Hurst exponent $H$ and its corresponding coefficient of determination $R^{2}$ are presented.

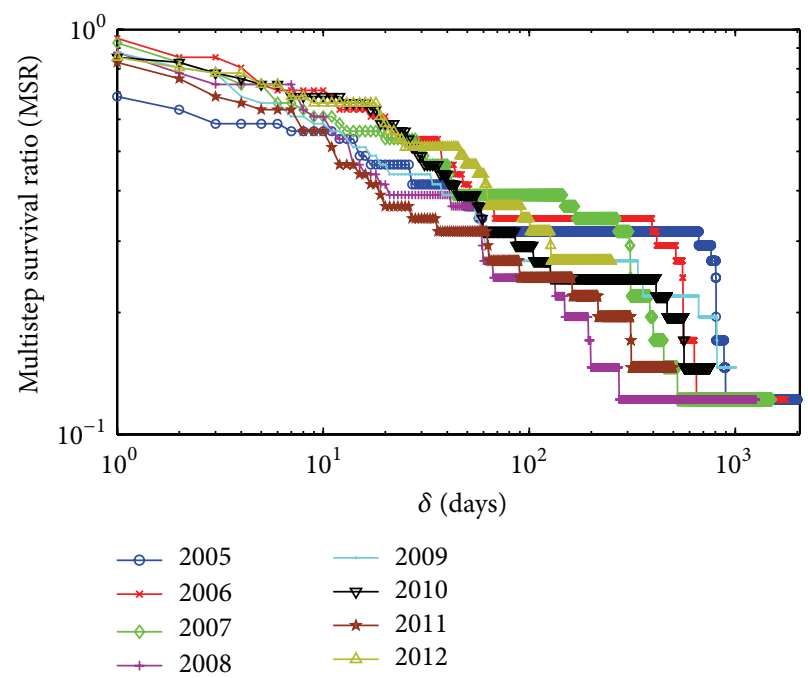

FIgURE 11: The multistep survival ratio (MSR) of MST of 42 currencies in the international FX market as a function of time for different initial time $t_{0}$. For each curve, the initial time $t_{0}$ is the first trading date of the year; for example, 2005 stands for January 5, 2005.

we employ the time-varying Student's $t$-copula to calculate the dynamic cross-correlation coefficients between each pair of rates. Finally we adopt the MST to build time-varying FX networks and analyze the networks properties including the dynamics and time correlations of topological features and survival rates of the MST.

Some basic finding for examining FX networks in this research can be summarized as follows. (i) By analyzing the descriptive statistics of cross-correlation coefficients and distances of MST, we find that distributions of cross-correlation coefficients (distances) in the international FX market (network) are fat-tailed and negatively skewed. (ii) On basis 
of MSTs for three different periods, we observe that some currencies gather together and form into several clusters, such as the international cluster with USD at its center, the Middle Eastern cluster, and the European cluster. The financial crises have a great influence on the FX network's topology structure and lead to USD becoming more centered in the MST because lots of currencies from Asia, Latin America, Middle East, and Africa are directly or indirectly linked to USD. (iii) The topological measures of the FX network present a large fluctuation and have a long-term memory effect. By estimating the degree distribution of MST, we find that the FX network is a scale-free network in most of the time. (iv) A great majority of links between currencies in the international FX market survive from one time to the next, and multistep survive rates descend sharply as the time increases.

\section{Conflict of Interests}

The authors declare that there is no conflict of interests regarding the publication of this paper.

\section{Acknowledgments}

The authors thank C. Yu who works in the Guosen Securities Co., Ltd., for helpful discussions. This work was supported by the Fundamental Research Funds for the Central Universities of Hunan University, the Hunan Provincial Innovation Foundation for Postgraduate (Grant no. CX2013A006), the Scholarship Award for Excellent Doctoral Student granted by the Ministry of Education of China, the National Natural Science Foundation of China (Grant no. 71373072), the Specialized Research Fund for the Doctoral Program of Higher Education (Grant no. 20130161110031), the China Postdoctoral Science Foundation (Grant no. 2013M530376), and the Foundation for Innovative Research Groups of the National Natural Science Foundation of China (Grant no. 71221001).

\section{References}

[1] R. N. Mantegna and H. E. Stanley, Introduction to Econophysics: Correlations and Complexity in Finance, Cambridge University Press, Cambridge, UK, 1999.

[2] J. Kwapień and S. Drożdż, "Physical approach to complex systems," Physics Reports, vol. 515, no. 3-4, pp. 115-226, 2012.

[3] C. Huang, C. Peng, X. Chen, and F. Wen, "Dynamics analysis of a class of delayed economic model," Abstract and Applied Analysis, vol. 2013, Article ID 962738, 12 pages, 2013.

[4] C. Huang, H. Kuang, X. Chen, and F. Wen, "An LMI approach for dynamics of switched cellular neural networks with mixed delays," Abstract and Applied Analysis, vol. 2013, Article ID 870486, 8 pages, 2013.

[5] R. N. Mantegna, "Hierarchical structure in financial markets," European Physical Journal B, vol. 11, no. 1, pp. 193-197, 1999.

[6] V. Boginski, S. Butenko, and P. M. Pardalos, "Statistical analysis of financial networks," Computational Statistics and Data Analysis, vol. 48, no. 2, pp. 431-443, 2005.
[7] J.-P. Onnela, K. Kaski, and J. Kertész, "Clustering and information in correlation based financial networks," European Physical Journal B, vol. 38, no. 2, pp. 353-362, 2004.

[8] M. Tumminello, T. Aste, T. Di Matteo, and R. N. Mantegna, "A tool for filtering information in complex systems," Proceedings of the National Academy of Sciences of the United States of America, vol. 102, no. 30, pp. 10421-10426, 2005.

[9] J.-P. Onnela, A. Chakraborti, K. Kaski, and J. Kertész, "Dynamic asset trees and portfolio analysis," European Physical Journal B, vol. 30, no. 3, pp. 285-288, 2002.

[10] J.-P. Onnela, A. Chakraborti, K. Kaski, J. Kertész, and A. Kanto, "Dynamics of market correlations: taxonomy and portfolio analysis," Physical Review E-Statistical, Nonlinear, and Soft Matter Physics, vol. 68, no. 5, Article ID 056110, 12 pages, 2003.

[11] J. G. Brida and W. A. Risso, "Dynamics and structure of the 30 largest North American companies," Computational Economics, vol. 35, no. 1, pp. 85-99, 2010.

[12] W.-Q. Huang, X.-T. Zhuang, and S. Yao, "A network analysis of the Chinese stock market," Physica A: Statistical Mechanics and Its Applications, vol. 388, no. 14, pp. 2956-2964, 2009.

[13] C. K. Tse, J. Liu, and F. C. M. Lau, "A network perspective of the stock market," Journal of Empirical Finance, vol. 17, no. 4, pp. 659-667, 2010.

[14] D. Y. Kenett, M. Tumminello, A. Madi, G. Gur-Gershgoren, R. N. Mantegna, and E. Ben-Jacob, "Dominating clasp of the financial sector revealed by partial correlation analysis of the stock market," PLoS ONE, vol. 5, no. 12, Article ID e15032, 2010.

[15] A. Z. Górski, S. Drożdż, and J. Kwapień, "Scale free effects in world currency exchange network," The European Physical Journal B, vol. 66, no. 1, pp. 91-96, 2008.

[16] J. Kwapień, S. Gworek, S. Drożdż, and A. Górski, "Analysis of a network structure of the foreign currency exchange market," Journal of Economic Interaction and Coordination, vol. 4, no. 1, pp. 55-72, 2009.

[17] J. Kwapień, A. Górski, and S. Drożdż, "Structure and evolution of the foreign exchange networks," Acta Physica Polonica B, vol. 40, no. 1, pp. 175-194, 2009.

[18] W. Jang, J. Lee, and W. Chang, "Currency crises and the evolution of foreign exchange market: evidence from minimum spanning tree," Physica A: Statistical Mechanics and Its Applications, vol. 390, no. 4, pp. 707-718, 2011.

[19] G.-J. Wang, C. Xie, F. Han, and B. Sun, "Similarity measure and topology evolution of foreign exchange markets using dynamic time warping method: evidence from minimal spanning tree," Physica A: Statistical Mechanics and Its Applications, vol. 391, no. 16, pp. 4136-4146, 2012.

[20] D. Matesanz and G. J. Ortega, "Network analysis of exchange data: Interdependence drives crisis contagion," Quality \& Quantity, 2013.

[21] G.-J. Wang, C. Xie, Y.-J. Chen, and S. Chen, "Statistical properties of the foreign exchange network at different time scales: evidence from detrended cross-correlation coefficient and minimum spanning tree," Entropy, vol. 15, no. 5, pp. 16431662, 2013.

[22] D.-M. Song, M. Tumminello, W.-X. Zhou, and R. N. Mantegna, "Evolution of worldwide stock markets, correlation structure, and correlation-based graphs," Physical Review E-Statistical, Nonlinear, and Soft Matter Physics, vol. 84, no. 2, Article ID 026108, 9 pages, 2011.

[23] Š. Lyócsa, T. Výrost, and E. Baumöhl, "Stock market networks: the dynamic conditional correlation approach," Physica A: 
Statistical Mechanics and Its Applications, vol. 391, no. 16, pp. 4147-4158, 2012.

[24] C. Huang, X. Gong, X. Chen, and F. Wen, "Measuring and forecasting volatility in Chinese stock market using HAR-CJM model," Abstract and Applied Analysis, vol. 2013, Article ID 143194, 13 pages, 2013.

[25] T. Trancoso, "Emerging markets in the global economic network: real(ly) decoupling?" Physica A: Statistical Mechanics and Its Applications, vol. 395, pp. 499-510, 2014.

[26] M. Sklar, "Fonctions de répartition à $n$ dimensions et leurs marges," Publications de l'Institut de Statistique de l'Université de Paris, vol. 8, pp. 229-231, 1959.

[27] A. Sklar, "Random variables, joint distribution functions, and copulas," Kybernetika, vol. 9, pp. 449-460, 1973.

[28] A. C. Cameron, T. Li, P. K. Trivedi, and D. M. Zimmer, "Modelling the differences in counted outcomes using bivariate copula models with application to mismeasured counts," The Econometrics Journal, vol. 7, no. 2, pp. 566-584, 2004.

[29] F. Wen and Z. Liu, "A copula-based correlation measure and its application in chinese stock market," International Journal of Information Technology \& Decision Making, vol. 8, no. 4, pp. 787-801, 2009.

[30] J. Hu, "Dependence structures in Chinese and US financial markets: a time-varying conditional copula approach," Applied Financial Economics, vol. 20, no. 7, pp. 561-583, 2010.

[31] A. J. Patton, "Estimation of multivariate models for time series of possibly different lengths," Journal of Applied Econometrics, vol. 21, no. 2, pp. 147-173, 2006.

[32] R. Aloui, M. S. Ben Aïssa, and D. K. Nguyen, "Conditional dependence structure between oil prices and exchange rates: a copula-GARCH approach," Journal of International Money and Finance, vol. 32, pp. 719-738, 2013.

[33] R. Aloui, S. Hammoudeh, and D. K. Nguyen, "A time-varying copula approach to oil and stock market dependence: the case of transition economies," Energy Economics, vol. 39, pp. 208-221, 2013.

[34] K. Wang, Y.-H. Chen, and S.-W. Huang, “The dynamic dependence between the Chinese market and other international stock markets: a time-varying copula approach," International Review of Economics and Finance, vol. 20, no. 4, pp. 654-664, 2011.

[35] A. J. Patton, "Modelling asymmetric exchange rate dependence," International Economic Review, vol. 47, no. 2, pp. 527556, 2006.

[36] C. Diks, V. Panchenko, and D. van Dijk, "Out-of-sample comparison of copula specifications in multivariate density forecasts," Journal of Economic Dynamics and Control, vol. 34, no. 9, pp. 1596-1609, 2010.

[37] A. Dias and P. Embrechts, "Modeling exchange rate dependence dynamics at different time horizons," Journal of International Money and Finance, vol. 29, no. 8, pp. 1687-1705, 2010.

[38] A. Clauset, C. R. Shalizi, and M. E. J. Newman, "Power-law distributions in empirical data," SIAM Review, vol. 51, no. 4, pp. 661-703, 2009.

[39] Y. Wei, Y. Wang, and D. Huang, "A copula-multifractal volatility hedging model for CSI 300 index futures," Physica A: Statistical Mechanics and Its Applications, vol. 390, no. 23-24, pp. 42604272, 2011.

[40] Y. Lai, C. W. S. Chen, and R. Gerlach, "Optimal dynamic hedging via copula-threshold-GARCH models," Mathematics and Computers in Simulation, vol. 79, no. 8, pp. 2609-2624, 2009.
[41] $\mathrm{H}$. Joe and J. J. Xu, "The estimation method of inference functions for margins for multivariate models," Tech. Rep. 166, Department of Statistics, University of British Columbia, Vancouver, Canada, 1996.

[42] J. B. Kruskal Jr., "On the shortest spanning subtree of a graph and the traveling salesman problem," Proceedings of the American Mathematical Society, vol. 7, pp. 48-50, 1956.

[43] C. Yang, Y. Shen, and B. Xia, "Evolution of Shanghai stock market based on maximal spanning trees," Modern Physics Letters B, vol. 27, no. 3, Article ID 135002, 19 pages, 2013.

[44] N. Vandewalle, F. Brisbois, and X. Tordoir, "Non-random topology of stock markets," Quantitative Finance, vol. 1, no. 3, pp. 372-374, 2001.

[45] R. Albert and A.-L. Barabási, "Statistical mechanics of complex networks," Reviews of Modern Physics, vol. 74, no. 1, pp. 47-97, 2002.

[46] T. Aste, W. Shaw, and T. Di Matteo, "Correlation structure and dynamics in volatile markets," New Journal of Physics, vol. 12, no. 8, Article ID 085009, 21 pages, 2010.

[47] T. Qiu, B. Zheng, and G. Chen, "Financial networks with static and dynamic thresholds," New Journal of Physics, vol. 12, no. 4, Article ID 043057, 16 pages, 2010.

[48] C.-K. Peng, S. V. Buldyrev, S. Havlin, M. Simons, H. E. Stanley, and A. L. Goldberger, "Mosaic organization of DNA nucleotides," Physical Review E, vol. 49, no. 2, pp. 1685-1689, 1994. 


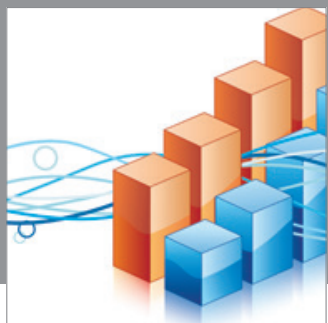

Advances in

Operations Research

mansans

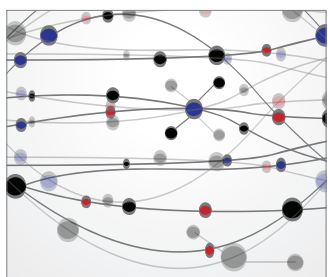

The Scientific World Journal
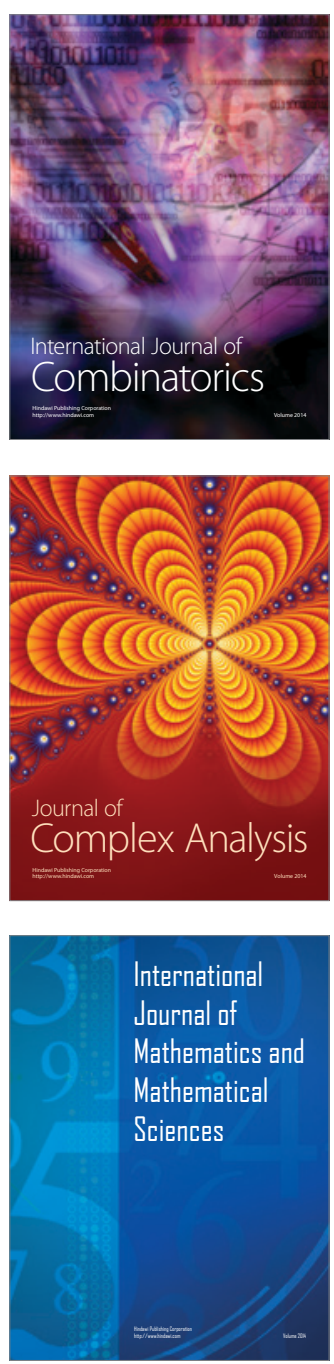
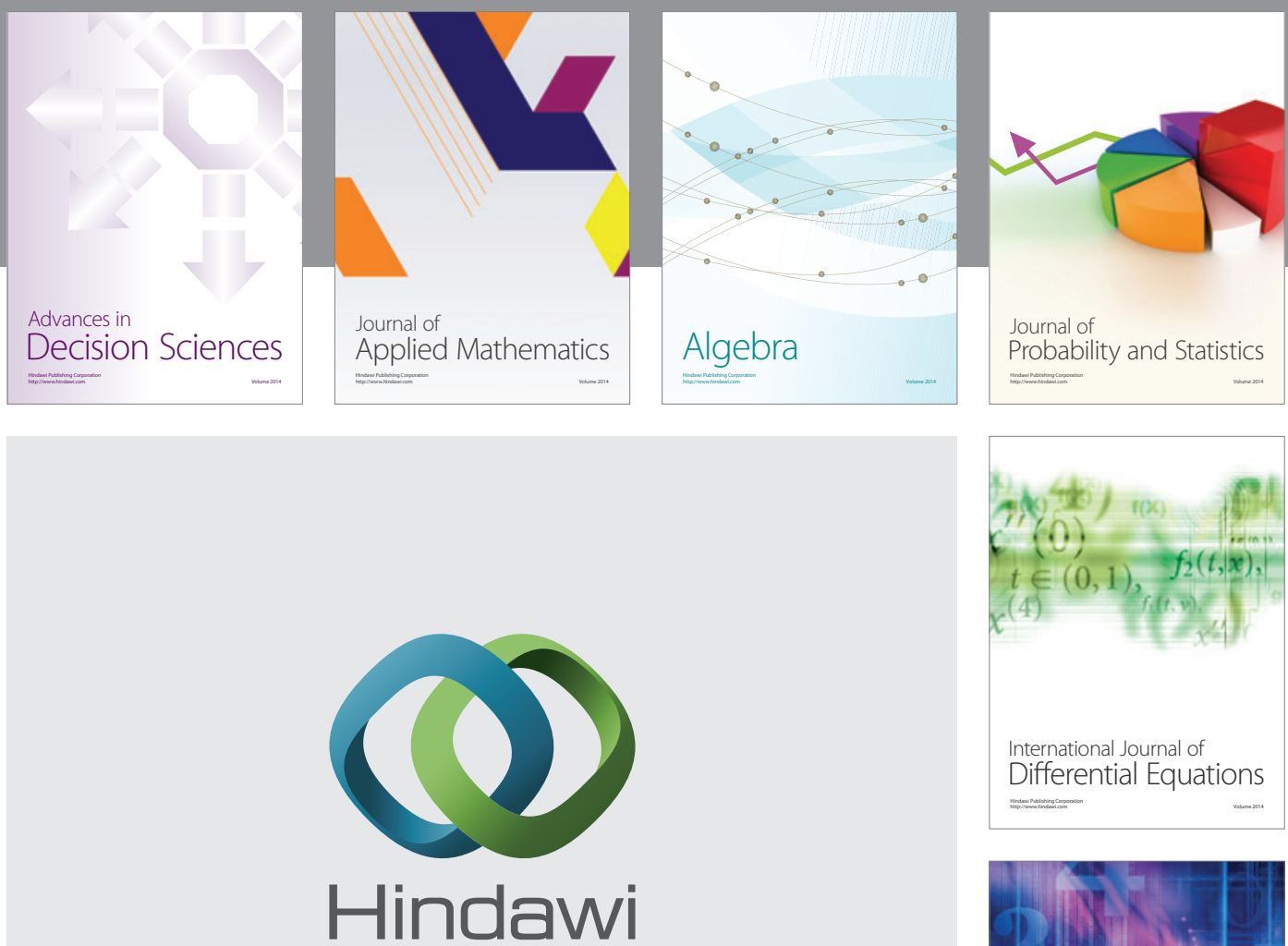

Submit your manuscripts at http://www.hindawi.com
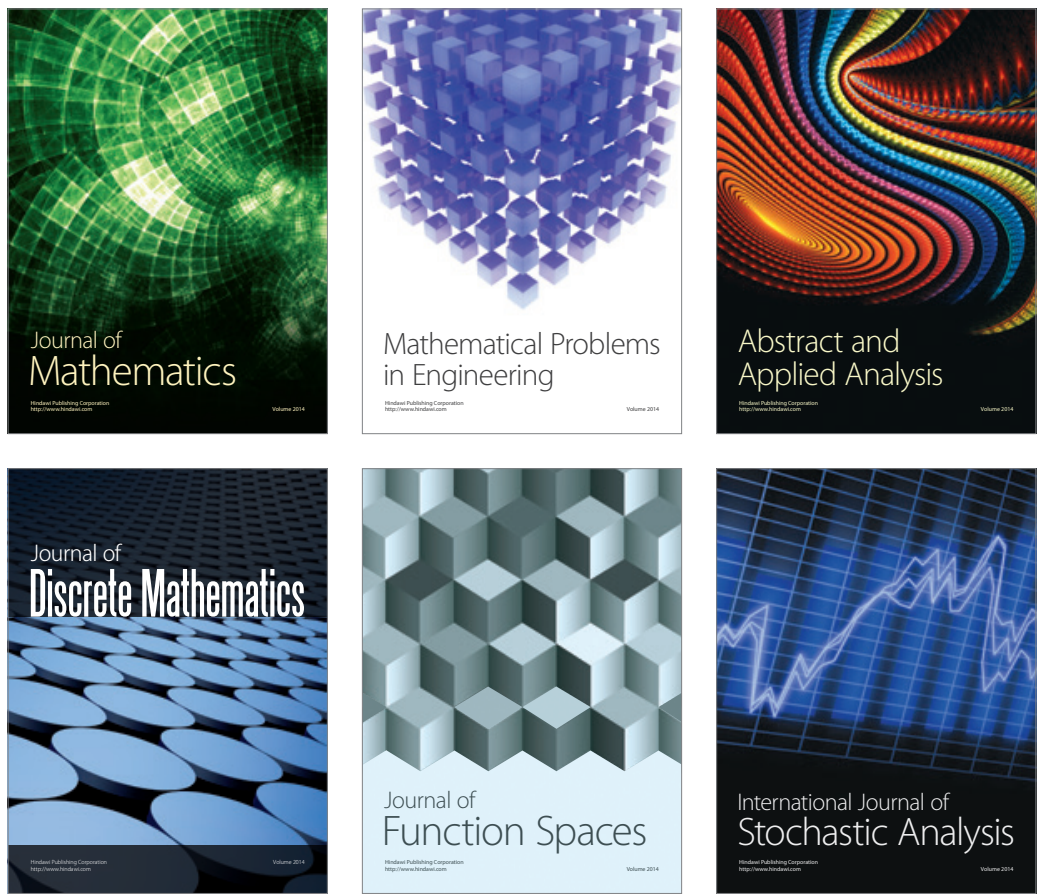

Journal of

Function Spaces

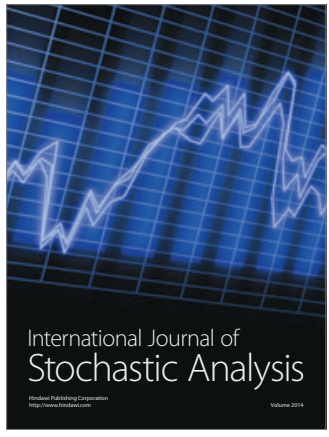

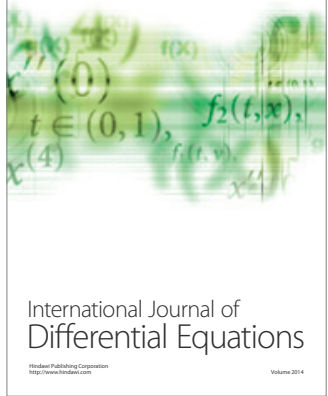
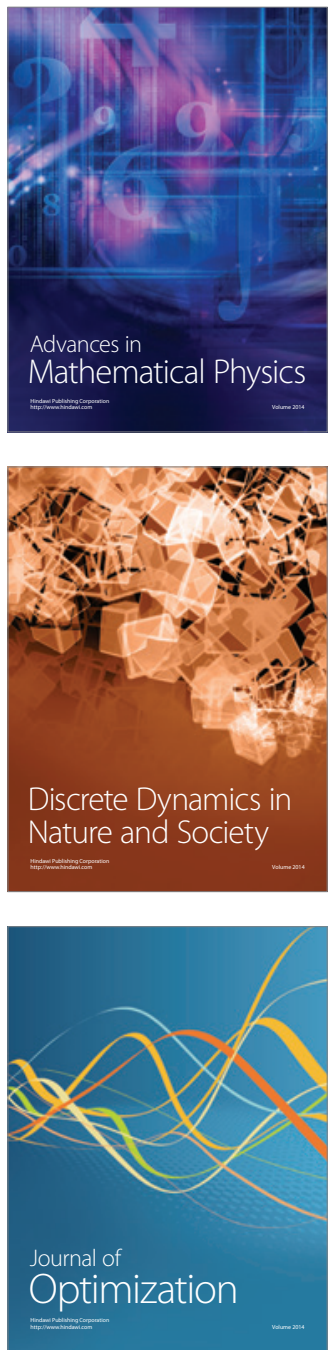